\title{
OPTICAL IDENTIFICATION OF SOUTHERN RADIO SOURCES
}

\author{
JANE BASINSKI, B. J. BOK, AND K. GOTTLIEB \\ Mount Stromlo Observatory, Canberra, Australia
}

The identification of discrete radio sources with optically observed celestial objects continues to be one of the most difficult and challenging tasks for the optical astronomer. Baade and Minkowski [1,2] have done admirable work on the subject for the northern hemisphere and for the sections of the southern hemisphere accessible from the latitudes of Mount Wilson and Palomar Observatories. The zone south of declination -40 degrees, however, has not been studied to any great extent. As a part of our collaborative program of research with the C.S.I.R.O. Radiophysics Laboratory in Sydney, several members of the Mount Stromlo Observatory staff have initiated studies for the identification of sources with declinations south of -40 degrees.

At present for this type of research we have available at Mount Stromlo Observatory the 74-inch reflector-with a primary mirror still affected by a slight amount of inherent astigmatism-and an $f / 1$ Meinel Pearson Schmidt camera. We have also, by courtesy of Professor K. G. Malmquist and Dr. B. E. Westerlund, limited access to the $f / 3.5$ Uppsala Schmidt telescope located at Mount Stromlo Observatory. To obtain fair definition over an area of $6 \frac{1}{2}$ by $6 \frac{1}{2}$ inches, we have used the 74 -inch telescope at the Newtonian focus stopped down to a 45-inch aperture; for our purposes the 74-inch reflector operates at $f / 8$. We do not yet possess a nebular spectrograph capable of yielding spectra for some of the filaments of nebulous material that may be related to sources of radio brightness.

The search for optical equivalents of discrete radio sources is a discouraging business, since, after much labor, so few positive results are obtained. There seems to be no alternative to careful photography in blue and red light of the region of each radio source. From Mills we obtained a list of radio sources of considerable intensity in the radio range, which appear to be the most promising candidates for identification. The present report is concerned with results from detailed inspection of photographs made with the 74-inch reflector and with the Uppsala Schmidt telescope. For each center we have also examined photographs in $\mathrm{H} \alpha$ light made with the 8-inch Schmidt by Rodgers and Campbell. For the 74-inch reflector, the red filters used were a perspex filter of an approximate range of $500 \AA$ centered on $\mathrm{H} \alpha$; for the Uppsala Schmidt telescope, filter RG 1; and for the 8-inch Schmidt a filter of Chance OR 1 glass $8.5 \mathrm{~mm}$ thick transmitting 52 per cent at $\mathrm{H} \alpha$ and none at $6400 \AA$.

Because of our present lack of suitable spectrographic equipment, no positive identifications can be announced. In several cases, however, we have located 
either bright nebulous filaments, or a peculiar galaxy, or a cluster of faint galaxies at, or very near, Mills's radio position.

In order to test our potentialities we have included in our list of positions to be photographed some at declinations north of -40 degrees, for which optical identifications have already been obtained. One of the strongest southern sources is Puppis A (IAU 08S4A), identified by Baade and Minkowski [1]. The loose, detached filaments they describe are readily seen on our 74inch photographs and they are also detectable on the Uppsala Schmidt plates.

We have examined three additional centers north of declination -40 degrees:

(1) Radio position (Mills); $\alpha(1960)=10^{\mathrm{h}} 08^{\mathrm{m}} 1 \pm 0 . \mathrm{m} 3 ; \delta(1960)=-11^{\circ} 50^{\prime} \pm 7^{\prime}$; $l=220^{\circ} ; b=+36^{\circ} ; S=30 \times 10^{-26}$ watts $\mathrm{m}^{-2}(\mathrm{c} / \mathrm{s})^{-1}$. Mills notes that the agreement with the position of NGC $3145, \alpha(1960)=10^{\mathrm{h}} 08^{\mathrm{m}} 1 ; \delta(1960)=-12^{\circ} 13^{\prime}$, is very poor. He states that the radio source is apparently an extended one with a diameter of one degree. If NGC 3145 is a radio emitter, then there is probably also a second source in the field, yet to be identified optically. Our photographs show NGC 3145 and also several faint galaxies near the radio center, but no further identification is suggested.

(2) Radio position (Mills); $\alpha(1960)=12^{\mathrm{h}} 00^{\mathrm{m}} 0 \pm 00^{\mathrm{m}} 2 ; \delta(1960)=-18^{\circ} 44^{\prime} \pm 6^{\prime}$; $l=256^{\circ} ; b=+43^{\circ} ; S=10 \times 10^{-26}$ watts $\mathrm{m}^{-2}(\mathrm{c} / \mathrm{s})^{-1}$. There is a complex galaxy NGC $4038 / 39$ at $\alpha(1960)=11^{\text {b } 59 \text { m } 9 ;} \delta(1960)=-18^{\circ} 38^{\prime} ;$ in addition, NGC 4027 is at $\alpha(1960)=11^{\mathrm{h}} 57^{\mathrm{m}} \cdot 6, \delta(1960)=-19^{\circ} 02^{\prime}$. This combination has been described in detail by Shapley and Paraskevopoulos [5], who stressed that the presence of two very peculiar but rather similar ringtail-shaped galaxies is not found anywhere else in the sky. Minkowski was apparently the first to suggest that the field might contain a strong radio-emitter, and Mills indeed found this to be the case-at least with regard to NGC 4038/39. Pawsey [4] has a reference to this center. Minkowski regards NGC 4038/39 as a pair of galaxies in collision. Our photographs show well the peculiar shapes of all three objects, but it is not clear from our material that NGC 4038/39 is a double galaxy rather than a single disturbed one. The Uppsala Schmidt telescope plates show, however, two nebulous arcs protruding from the combination, which may support the hypothesis of two galaxies in collision. A most remarkable feature of NGC $4038 / 39$ and 4027 is the presence of several very distinct and bright knots of nebulous material.

(3) Radio position (Mills); $\alpha(1960)=21^{\mathrm{h}} 59^{\mathrm{m}} 0 \pm 00^{\mathrm{m}} 4 ; \delta(1960)=-13^{\circ} 27^{\prime} \pm 5^{\prime}$; $l=13^{\circ} ; b=-49^{\circ} ; S=14 \times 10^{-26}$ watts $\mathrm{m}^{-2}(\mathrm{c} / \mathrm{s})^{-1}$. Mills notes that identification with NGC 7171, $\alpha(1960)=21^{\mathrm{h}} 58^{\mathrm{m}} 7 ; \delta(1960)=-13^{\circ} 27^{\prime}$ is highly probable. NGC 7171 appears on our photographs to be a normal spiral. There are several faint galaxies within the area of uncertainty, but there is no indication of a cluster of galaxies near the radio position.

The table gives the information regarding the centers south of declination -40 degrees examined in our survey, with, in the final column, the information given us by Mills. In the first column is the number of each center used for reference below.. The second and fourth columns show Mills's radio position, and the third and fifth columns give the estimated probable error according to Mills. The galactic latitude and longitude are shown in columns six and seven and the radio intensity of the source is in the eighth column. 
Radio Data and Notes

\begin{tabular}{|c|c|c|c|c|c|c|c|}
\hline Center & $\alpha(1960)$ & $\Delta \alpha$ & $\delta(1960)$ & $\Delta \delta$ & $l$ & $b$ & $\underset{\text { watts } \mathrm{m}^{-2}(\mathrm{c} / \mathrm{s})^{-1}}{S}$ \\
\hline 1 & $02^{\mathrm{h}} 42^{\mathrm{m} 3}$ & $\pm 0 \mathrm{~m} 2$ & $-51^{\circ} 19^{\prime}$ & $\pm 3^{\prime}$ & $234^{\circ}$ & $-58^{\circ}$ & $41 \times 10^{-20}$ \\
\hline 2 & $04^{\mathrm{h}} 28^{\mathrm{m}} 0$ & $\pm 0 \mathrm{~m} 2$ & $-53^{\circ} 58^{\prime}$ & $\pm 3^{\prime}$ & $229^{\circ}$ & $-42^{\circ}$ & $58 \times 10^{-28}$ \\
\hline $3^{a}$ & $05^{\mathrm{h}} 18^{\mathrm{m}} 6$ & $\pm 0 \mathrm{~m} 1$ & $-45^{\circ} 48^{\prime}$ & $\pm 2^{\prime}$ & $219^{\circ}$ & $-34^{\circ}$ & $570 \times 10^{-20}$ \\
\hline $4^{b}$ & $08^{\mathrm{h}} 34^{\mathrm{m}} 0$ & $\pm 0 \mathrm{~m} 3$ & $-45^{\circ} 39^{\prime}$ & $\pm 4^{\prime}$ & $230^{\circ}$ & $-2^{\circ}$ & $1000 \times 10^{-20}$ \\
\hline $5^{c}$ & $12^{\mathrm{h}} 47^{\mathrm{m}} 1$ & $\pm 0 \mathrm{~m} 2$ & $-41^{\circ} 05^{\prime}$ & $\pm 3^{\prime}$ & $271^{\circ}$ & $+21^{\circ}$ & $25 \times 10^{-26}$ \\
\hline $6 d$ & $13^{h} 43 m 7$ & & $-60^{\circ} 15^{\prime}$ & & $277^{\circ}$ & $+1^{\circ}$ & \\
\hline $7 e$ & $16^{\mathrm{h}} 11 \mathrm{~m} 1$ & $\pm 0 \mathrm{~m} 2$ & $-60^{\circ} 54^{\prime}$ & $\pm 3^{\prime}$ & $293^{\circ}$ & $-8^{\circ}$ & \\
\hline 8 & $23^{\mathrm{h}} 56^{\mathrm{m}} 8$ & $\pm 0 \mathrm{~m} 2$ & $-61^{\circ} 06^{\prime}$ & $\pm 3^{\prime}$ & $280^{\circ}$ & $-56^{\circ}$ & $285 \times 10^{-20}$ \\
\hline
\end{tabular}

Center 1.-There are numerous faint galaxies in this field, but they show no evidence of clustering. No identification is suggested.

Center 2.- There is a marked cluster of galaxies at the radio position given by Mills, but some galaxies are also present in the remainder of the field. Close to the radio position there is a patch of nebulosity-or possibly an irregular galaxy-that requires further study. This patch is shown clearly on $103 \mathrm{aO}$ and $103 \mathrm{aE}$ plates without filter taken with the 74 -inch reflector. It it also shown on a $103 \mathrm{aE}$ plate with RG 1 filter taken with the Uppsala Schmidt telescope. IC 2082 is a possible identification suggested by Shapley (private communication to Mills).

Center 3.-There are several faint galaxies near the radio position, but otherwise nothing of interest is shown on our photographs. No identification suggested.

Center 4. - The region is very rich in unusual filamentary nebulosity, which covers an area of the sky of about 4 by 2 degrees (Gum [3]) and is notable for its unusually well-defined fronts and other sharp features. The nebulosity near this center bears no resemblance to the detached filaments observed near Puppis A. One fairly conspicuous detached filament can be seen approximately $3^{\mathrm{m}}$ in right ascension from the listed radio center, and one very faint filament close to the center. The latter is present on the $103 \mathrm{aO}$ and $103 \mathrm{aE}$ plates taken with the 74-inch reflector and also on the 103aE with RG 1 filter taken with the Uppsala Schmidt telescope.

Center 5. A rich cluster of bright galaxies is located at the radio position. No identification with a particular galaxy or pair of galaxies is suggested. NGC 4696, $\alpha(1960)=12^{\mathrm{b}} 46^{\mathrm{m}} 7, \delta(1960)=-41^{\circ} 05^{\prime}$ is close to the radio position. De Vaucouleurs [6] says of this cluster that it is "of outstanding density."

Center 6.-An interesting nebulous object is shown 25 minutes of arc $\mathrm{S}$ and $2^{\mathrm{m}} \mathrm{W}$ of the radio position. It is too far from the radio center to be accepted as an optical identification, but it may be the visible manifestation of a more extensive abnormal region. The object is $\mathrm{T}$-shaped and has a diameter of about one minute of arc. It is shown clearly on the 74-inch $103 \mathrm{aE}$ and $103 \mathrm{aE}$ with red filter plates, and faintly on the $103 \mathrm{aO}$ plates. It 
NO. 93

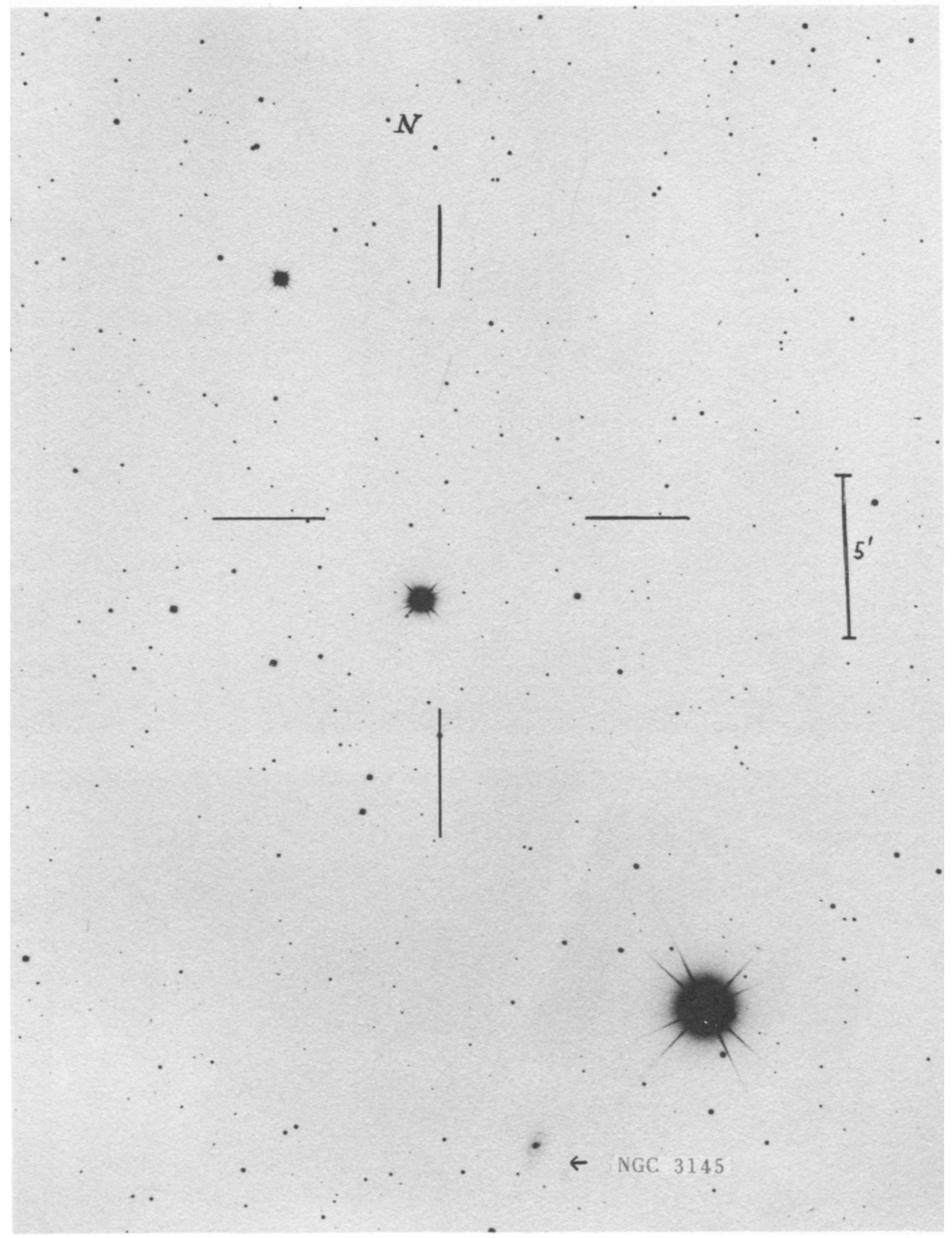

FIG. 1. Center $(a)$. The structure of NGC 3145 is clearly shown. Several faint galaxies are near the radio position. 103aE plate of 45 minutes' exposure. Mount Stromlo 74-inch reflector. On this and following figures the scale is given by a line segment 5 minutes of arc in length. 


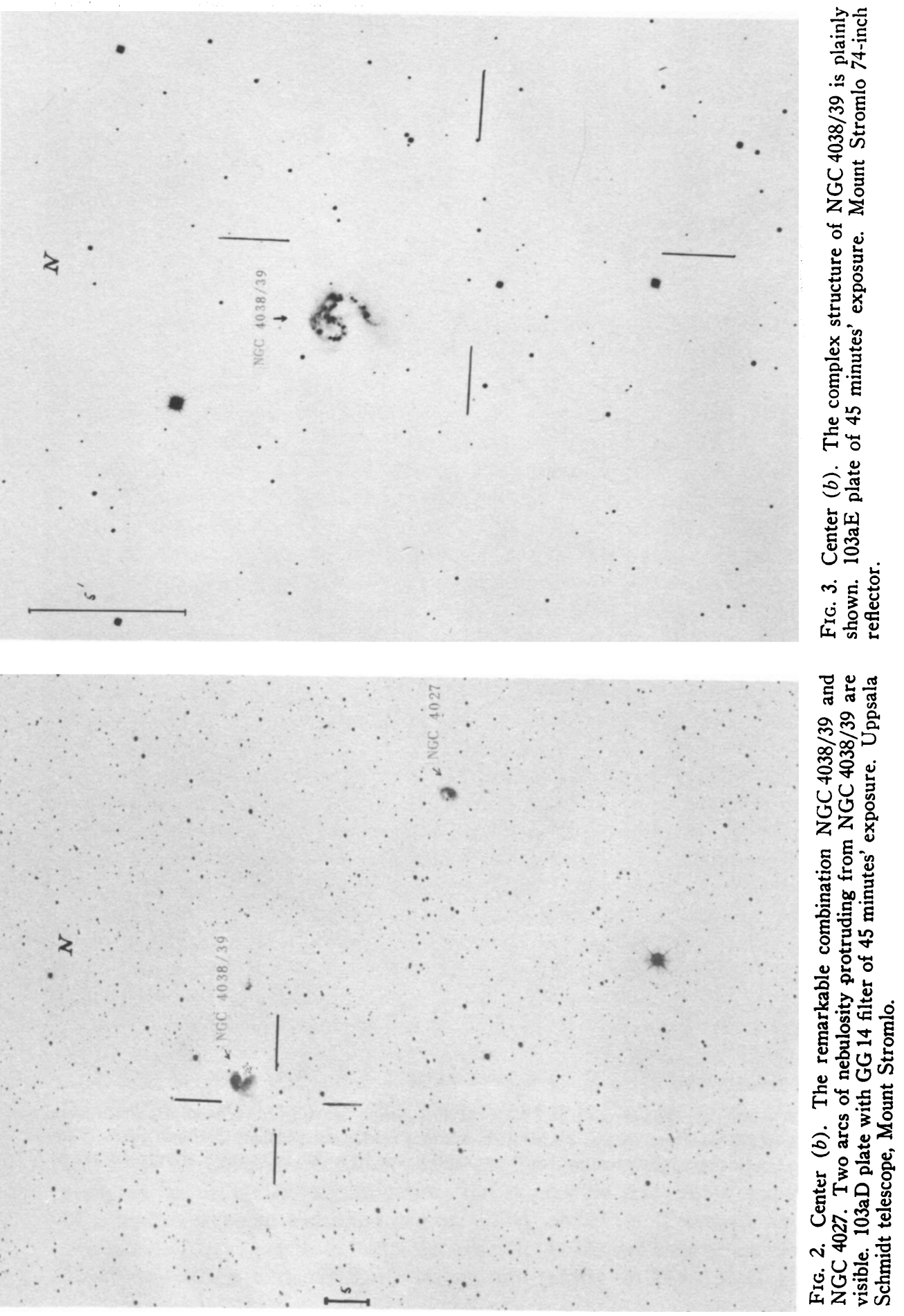



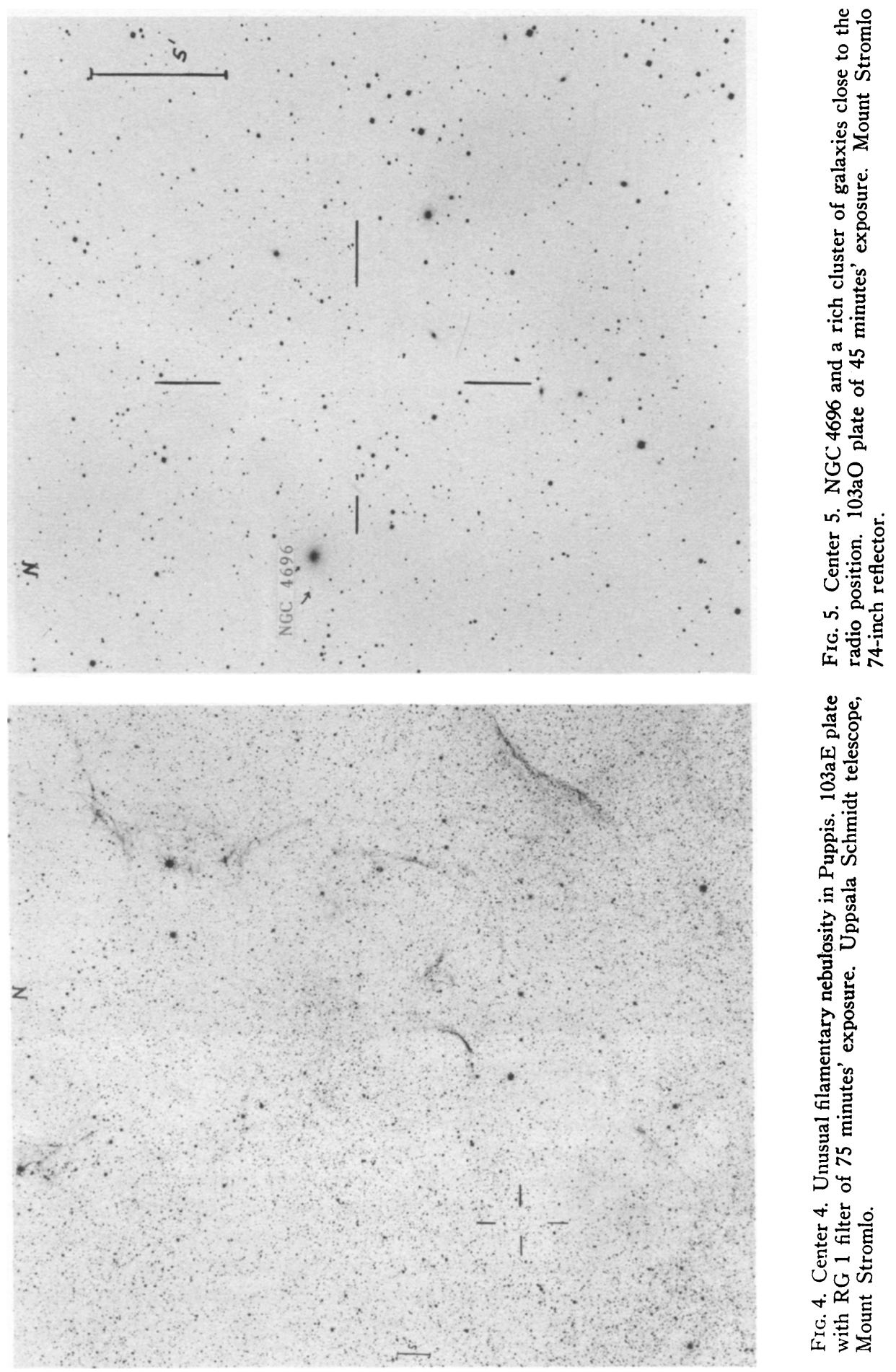


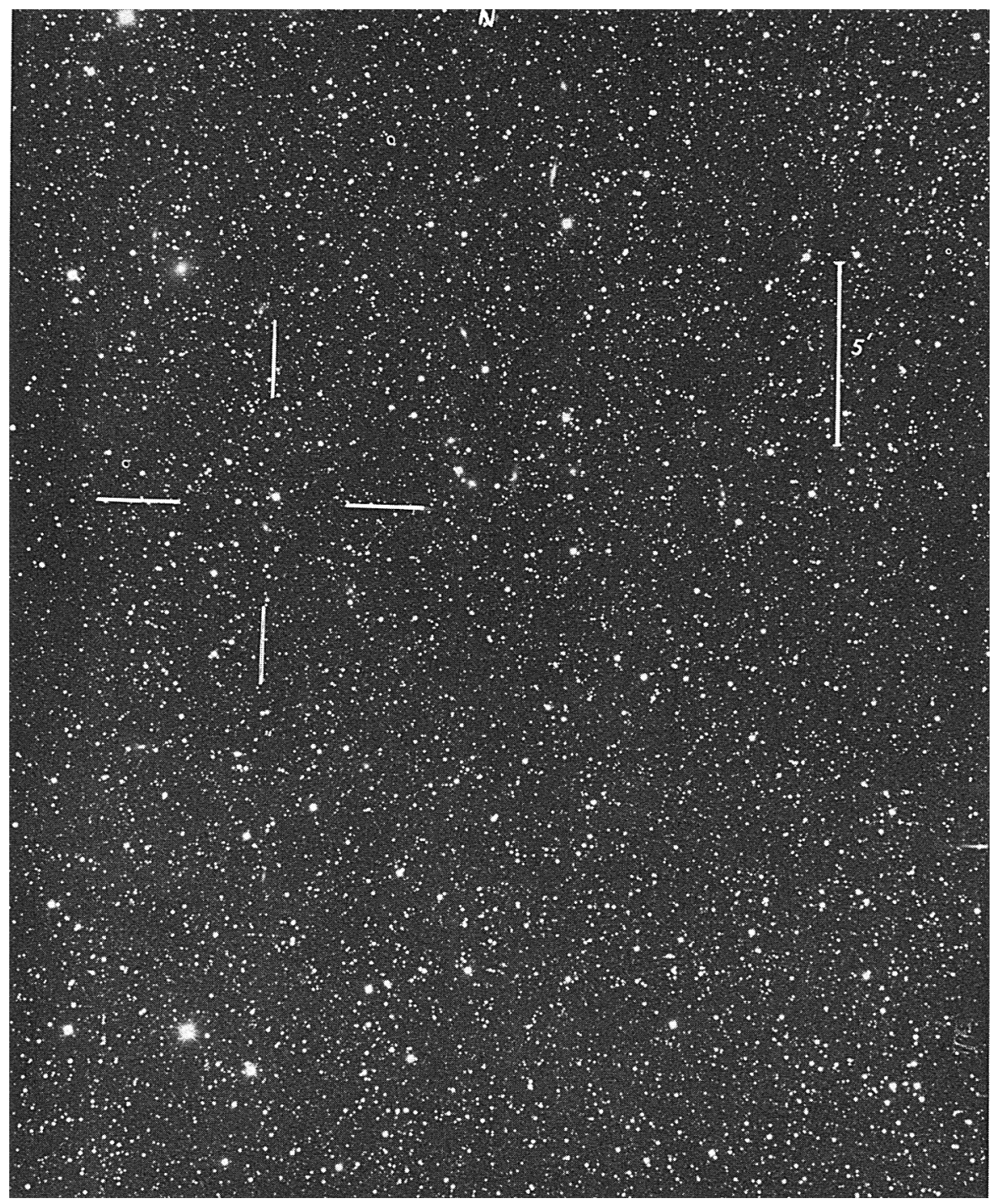

Fig. 6. Center 7. A field with numerous bright galaxies, many close to the radio center. $103 \mathrm{aE}$ plate of 60 minutes' exposure. Mount Stromlo 74-inch reflector. 


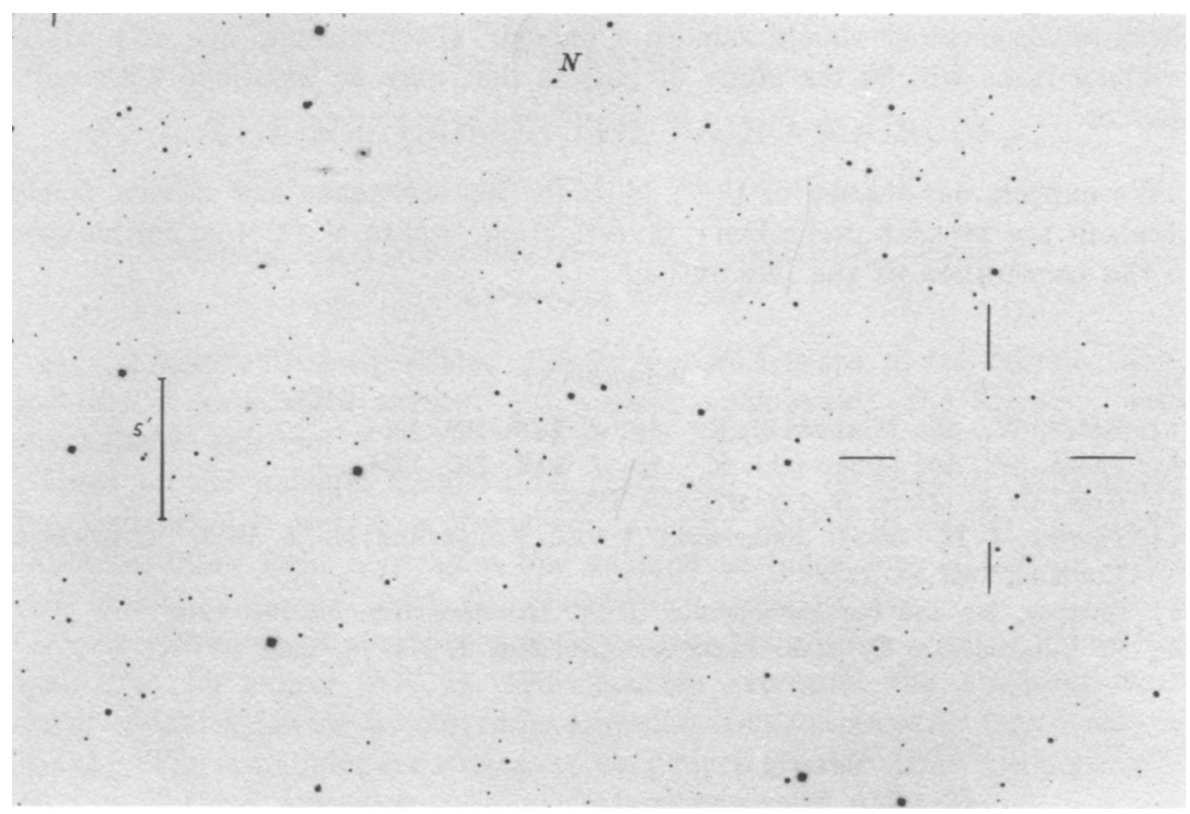

FIG. 7. Center 8. Many faint galaxies can be seen near the radio position. One conspicuous pair is approximately 3 minutes $\mathrm{W}$ of the radio center. $103 \mathrm{aO}$ plate of 45 minutes' exposure. Mount Stromlo 74-inch reflector.

is readily identified on Uppsala Schmidt photographs, $103 \mathrm{aE}$ without filter and $103 \mathrm{aE}$ with RG 1 filter. There is little nebulosity elsewhere in the field, and this "ghostlike" nebulous image is quite unusual.

Center 7.- The region is, for its low latitude, unusually rich in galaxies, and the radio center itself falls on a dense cluster of galaxies. No identification with any particular galaxy is suggested.

Center 8.-A number of faint galaxies are shown on the 74-inch photographs, and there appears to be a small cluster of them close to the radio position. A conspicuous pair of fairly bright galaxies can be seen about $3^{\mathrm{m}} \mathrm{W}$ of the radio position, but these occur well outside the limits set by Mills. No identification is suggested.

The foregoing cases show clearly the possibilities and the limitations for research in this field at Mount Stromlo Observatory. To supplement our existing auxiliary equipment, we hope to have available before long a MeinelCassegrain Field Corrector $(f / 2$ and $f / 5)$ for use with the 50 -inch reflector. This instrument should assist us in a variety of ways: first, in the photographic study of nebulous filaments and of faint galaxies, and clusters of them; second, for the detection of $\mathrm{H} \alpha$ emission objects through the interpolation of a transmission grating in the part of the instrument for which we shall have parallel light. Photoelectric studies of colors and magnitudes of faint galaxies of special interest are also a possibility. Before too many years, Mount 
Stromlo Observatory should acquire a nebular spectrograph, and one of its primary tasks will be the study of objects that may be identified with radio sources.

We express our thanks to B. Y. Mills for his assistance and advice freely given in the present preliminary investigation, and to V.O. Hunt for his care in the preparation of the illustrations.

\section{REFERENCES}

[1] Baade, W., and Minkowski, R. Ap. J. 119, 206, 1954.

[2] Baade, W., and Minkowski, R. Ap. J. 119, 215, 1954.

[3] Gum, C. S. Mem. R.A.S. 67, 155, 1955.

[4] Pawsey, J. L. Radio Astronomy (I.A.U. Symposium No. 4, 1955). Cambridge, England, 1957, p. 123.

[5] Shapley, H., and Paraskevopoulos, J. S. Harvard Reprint 184, 1940.

[6] de Vaucouleurs, G. Occ. Notes R. Astr. Soc. 3, No. 18, 1956. 